\title{
Phase matters: responding to and learning about peripheral stimuli depends on hippocampal $\theta$ phase at stimulus onset
}

\author{
Miriam S. Nokia, ${ }^{1}$ Tomi Waselius, ${ }^{1}$ Jarno E. Mikkonen, ${ }^{1,2}$ Jan Wikgren, ${ }^{1,2}$ \\ and Markku Penttonen ${ }^{1}$ \\ ${ }^{1}$ Department of Psychology, University of Jyvaskyla, Fl-40014 Jyväskylän, Finland; ${ }^{2}$ Centre for Interdisciplinary \\ Brain Research, University of Jyvaskyla, Fl-40014 Jyväskylän, Finland
}

\begin{abstract}
Hippocampal $\theta(3-12 \mathrm{~Hz})$ oscillations are implicated in learning and memory, but their functional role remains unclear. We studied the effect of the phase of local $\theta$ oscillation on hippocampal responses to a neutral conditioned stimulus (CS) and subsequent learning of classical trace eyeblink conditioning in adult rabbits. High-amplitude, regular hippocampal $\theta$-band responses (that predict good learning) were elicited by the CS when it was timed to commence at the fissure $\theta$ trough (Trough group). Regardless, learning in this group was not enhanced compared with a yoked control group, possibly due to a ceiling effect. However, when the CS was consistently presented to the peak of $\theta$ (Peak group), hippocampal $\theta$-band responding was less organized and learning was retarded. In well-trained animals, the hippocampal $\theta$ phase at CS onset no longer affected performance of the learned response, suggesting a time-limited role for hippocampal processing in learning. To our knowledge, this is the first study to demonstrate that timing a peripheral stimulus to a specific phase of the hippocampal $\theta$ cycle produces robust effects on the synchronization of neural responses and affects learning at the behavioral level. Our results support the notion that the phase of spontaneous hippocampal $\theta$ oscillation is a means of regulating the processing of information in the brain to a behaviorally relevant degree.
\end{abstract}

$\theta$ oscillation (3-12 Hz) (for review, see Buzsáki and Moser 2013) characterizes hippocampal local-field potentials in awake animals. Its amplitude is strongest near the hippocampal fissure, and its phase reverses between the hippocampal fissure/dentate gyrus and the CA1 pyramidal layer (Buzsáki 2002). $\theta$ is thought to reflect a state in which information about the surroundings is actively acquired (Buzsáki 1989). Animals exhibiting dominant hippocampal $\theta$ during spontaneous recordings prior to training tend to learn better (Berry and Thompson 1978; Nokia et al. 2009,2012 b), and high-amplitude, well phase-locked $\theta$-band hippocampal responses to the conditioned stimulus early in eyeblink conditioning predict good learning (Nokia et al. 2009, 2010, 2012a; Nokia and Wikgren 2014). Training contingent on transient episodes of hippocampal $\theta$ or its explicit absence has produced significant but somewhat inconsistent effects on learning (Griffin et al. 2004; Nokia and Wikgren 2014). In summary, both spontaneous and evoked hippocampal $\theta$ oscillations are connected to simple associative learning. Yet the mechanism behind these effects remains largely unknown.

According to a computational model by Hasselmo and colleagues (Hasselmo et al. 2002; Hasselmo and Stern 2014), the phase of hippocampal $\theta$ oscillation determines specific and separate time windows for the efficient encoding and retrieval of memories. During the trough of the fissure $\theta$ cycle, the hippocampus preferentially processes input arriving from multimodal cortical areas through the entorhinal cortex, leading to long-term potentiation (LTP) at the CA3-CA1 synapses (Huerta and Lisman 1995; Holscher et al. 1997; Hyman et al. 2003), while CA1 output back to the neocortex is suppressed (Kamondi et al.

Corresponding author: miriam.nokia@jyu.fi

Article is online at http://www.learnmem.org/cgi/doi/10.1101/lm.038166.115. Freely available online through the Learning \& Memory Open Access option.
1998). This, according to the model, supports the encoding of new information. Conversely, stimulation at the peak of fissure $\theta$ leads to depotentiation at the CA3 to CA1 synapses (Huerta and Lisman 1995; Holscher et al. 1997; Hyman et al. 2003) and to the firing of CA1 pyramidal cells (Skaggs et al. 1996; Kamondi et al. 1998), thereby relaying information processed in the hippocampus back to the entorhinal cortex. According to the model, the retrieval of already encoded information is favored at the peak of fissure $\theta$. A recent study suggested that electrical stimulation of the hippocampus at the $\theta$ trough and peak enhances the encoding and retrieval, respectively, of a spatial memory (Siegle and Wilson 2014). Based on the above discussion, the timing of intracranial stimulation relative to the local $\theta$ phase, at the very least, affects its consequences for hippocampal synaptic plasticity and output at the cellular and possibly also at the behavioral level. Whether $\theta$ phase also relates to learning about and responding to peripheral stimuli remains elusive.

Here, we studied whether the ongoing $\theta$ phase affects the hippocampal processing of peripheral stimuli and, if so, whether such effects carry over into learning. An overview of the experiment is presented in Figure 1A. First, we aimed to determine whether hippocampal responses to a neutral peripheral stimulus are modulated by $\theta$ phase. Female rabbits were implanted with recording electrodes in the hippocampus (see Fig. 1B), and, during a single session, presented with a 200-msec tone either during periods of spontaneously occurring hippocampal $\theta$ activity or irrespective of neural state. We expected to see better phase-locked hippocampal $\theta$-band responses when the tone onset overlapped with the fissure $\theta$ trough compared with when it started at $\theta$ peak. Next, we 
A

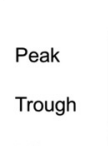

YC

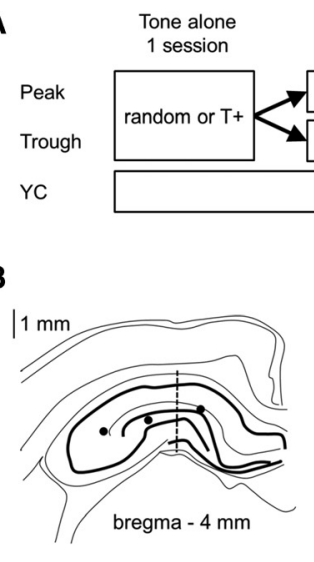

B
C

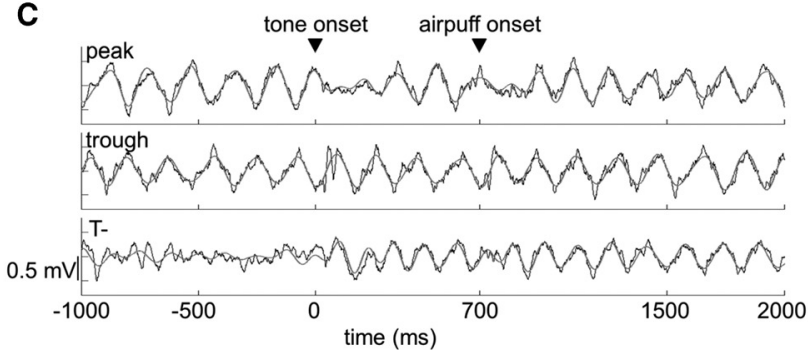

Figure 1. The effects of the phase (peak versus trough) of hippocampal fissure $\theta$ oscillation on hippocampal responding and trace eyeblink classical conditioning (EBCC) were studied $(A)$. Local-field potentials (LFPs) recorded from near the hippocampal fissure $(B)$ were used to trigger trials during trace eyeblink conditioning $(C)$. $(A)$ Adult female rabbits were divided into three groups. For animals in the Peak group $(n=6)$, the tone alone was presented either contingent on $\theta(T+)$ or irrespective of neural state. This was followed by trace eyeblink conditioning contingent on the peak of the $\theta$ oscillation (peak) and then contingent on the absence of $\theta(T-)$. Finally, the effect of $\theta$ phase on memory retrieval in this group was tested by presenting conditioning trials both to the peak and trough of the $\theta$ oscillation (peak and trough). For animals in the Trough group $(n=5)$, treatment was identical to that in the Peak group, except during the first 10 conditioning sessions, trials were presented contingent on the trough of the $\theta$ oscillation (trough). The yoked control group (YC, $n=6$ ) was trained irrespective of their neural state (random) at all times. (B) Representative example of the approximate placement of a linear probe (dashed line) used in recording hippocampal LFPs. Exact locations of single electrodes used for detecting $\theta$ in three animals are marked with filled circles. (C) Examples of hippocampal fissure LFPs prior to and during the presentation of the tone (conditioned stimulus) and the air puff (unconditioned stimulus). The top and middle panels depict single-trial LFPs in representative animals in the Peak (top) and Trough (middle) groups recorded during the first session of conditioning (beginning of "Trace EBCC 10 sessions" shown in $1 A$ ). The bottom panel in $C$ represents LFP from the same animal as in the top panel (peak) from session 11 (beginning of "Trace EBCC 6 sessions" in $A$ ). Note that now the conditioned stimulus was triggered by the absence of $\theta(T-)$. In $C$, the raw LFPs are printed in black and the $\theta$-band $(4-8 \mathrm{~Hz})$ filtered signal in gray. Arrowheads indicate the onset of the 200 -msec tone used as a conditioned stimulus and the onset of the 100-msec air puff toward the eye used as an unconditioned stimulus.

studied whether the phase of the $\theta$ oscillation at stimulus onset might have effects on learning about that stimulus. To this end, the rabbits were trained for 10 sessions in trace eyeblink classical conditioning, with the conditioned stimulus (CS, the same tone as before) timed to start at either the peak (Peak group) or trough (Trough group) of the fissure $\theta$ cycle (see Fig. 1C). Yoked control (YC) animals were trained simultaneously and received trials irrespective of their neural state. We expected higher phase-locking of hippocampal $\theta$-band responses to the CS in the Trough compared with the Peak group, and better learning in the Trough group compared with both the Peak and the YC groups. We then conditioned all the rabbits for another six sessions to maximize the number of animals that learned. Now, animals previously trained contingent on $\theta$ phase were trained in the explicit absence of $\theta$ ( $\mathrm{T}-$ ) because in our previous study (Nokia and Wikgren 2014) it led to a greater proportion of animals learning compared with random presentation of training trials. Animals in the YC group continued to be trained irrespective of their neural state. We expected animals in the experimental groups (Peak and Trough) to learn the task during the $\mathrm{T}$ - training, if they had not already done so. Last, to study the effects of $\theta$ phase on memory retrieval, we presented conditioning trials to well-learned animals: A total of two sessions were conducted, each with 30 trials to the peak and 30 to the trough of $\theta$, in random order. Better memory retrieval, that is, a higher number of learned responses, was expected when the CS was presented to the peak of the fissure $\theta$ cycle compared with when it was presented to the trough of the $\theta$ cycle. and its phase reverses between the CA1 pyramidal layer and the fissure/dentate gyrus. Curiously, in rabbits, the phase of $\theta$ oscillation remains constant across the fissure, the dentate gyrus and the hilus. Note that in rats, comparison of the dentate gyrus and the hilus also shows a phase-shift in $\theta$ (Buzsáki et al. 1983).

\section{Hippocampal $\theta$-band responses to a neutral tone were modulated by ongoing, spontaneous $\theta$ oscillations}

During a single session conducted prior to any conditioning, all the rabbits were presented with a $200-\mathrm{msec}, 80-\mathrm{dB}, 4-\mathrm{kHz}$ tone. For the animals later assigned to the experimental groups, the tone was presented 300 times either during periods of spontaneously occurring hippocampal $\theta$ activity $(\mathrm{T}+, n=5)$ or irrespective of neural state (random, $n=6$ ). For the animals assigned to the YC group $(n=6)$ for reasons of technical difficulties in obtaining neural recordings, stimuli were presented irrespective of neural state. This session was carried out to find out if $\theta$ phase, and the neural state at large ( $\mathrm{T}+$ versus random), affects $\theta$-band hippocampal responses to a neutral peripheral stimulus. By $\theta$-band responses we refer to responses occurring at the frequency band of $4-8 \mathrm{~Hz}$. Phase-locking and relative amplitude, quantified as the $\theta$ ratio (\%) for these responses, is analyzed separately. For details on these measures, please see Materials and Methods.

First we analyzed the effects of the preceding neural state $(\mathrm{T}+$ versus random) on the relative amplitude and phase-locking of the hippocampal fissure $\theta$-band responses to the neutral tone 
A

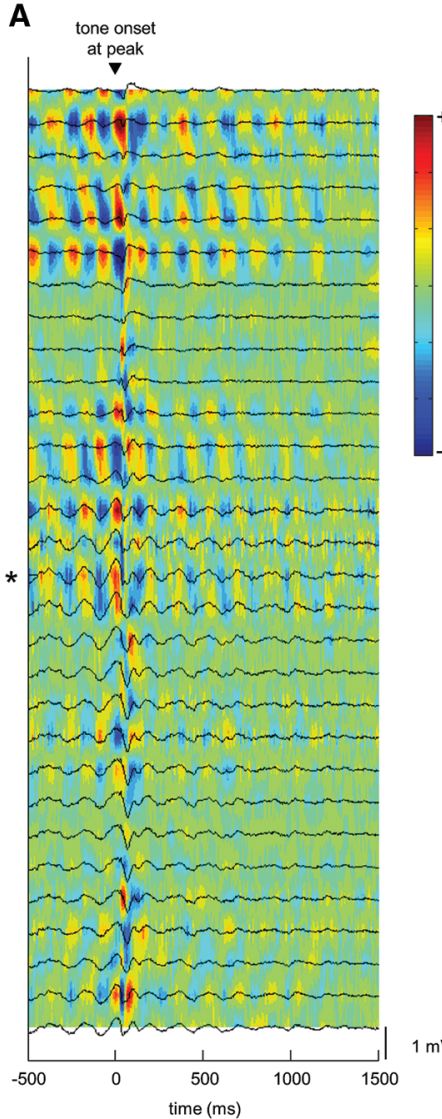

B

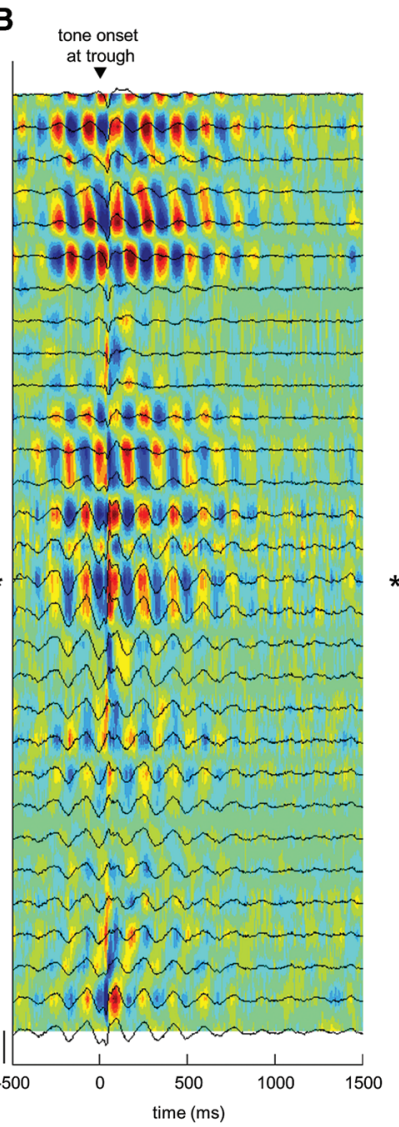

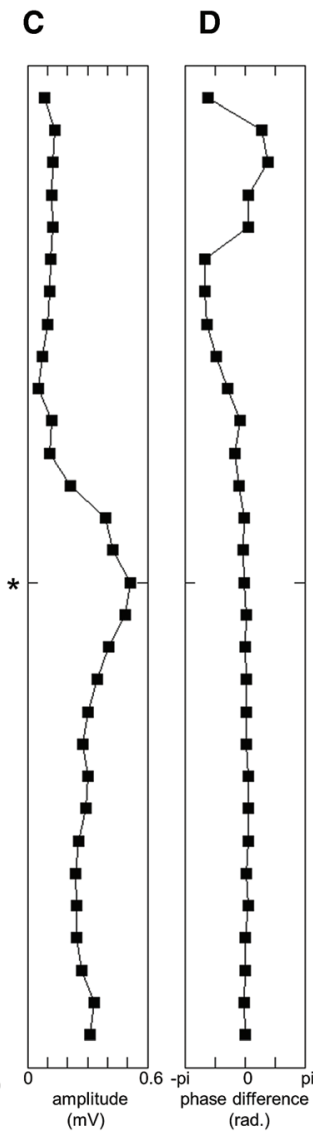

the ongoing $\theta$ oscillation at tone onset. However, as hypothesized, better phaselocked responses were elicited when the tone onset fell at the trough of the $\theta$ cycle $(0.53 \pm 0.05)$ compared with the peak of the $\theta$ cycle $(0.29 \pm 0.05)$. This effect was evident in four out of the five animals. Figure 2 depicts examples of event-related averages and current source density plots (please see Materials and Methods) of local-field potentials (LFPs) recorded from the hippocampus with a linear probe in response to the tone alone when it commenced at the peak (A) or the trough (B) of the fissure $\theta$ cycle in one representative animal. Visual comparison of A and B suggests better phase-locking of $\theta$-band responses to the tone when it was presented to $\theta$ trough compared with $\theta$ peak. Although the effect was not quite statistically significant at a group level $\left[t_{(4)}=2.62, P=0.059\right]$, we carried on with the experiment as planned.

\section{Baseline responses to the tone, spontaneous $\theta$ ratios and intertrial intervals did not differ between groups}

The 11 animals with functional recording electrodes were assigned to two experimental groups (Peak, $n=6$ versus Trough, $n=5$ ) for subsequent trace eyeblink conditioning. Half the animals in the Peak and Trough groups had, prior to conditioning, received tone-alone presentations to a random brain state and half during $\theta$. There was no statistically significant difference between the Peak and Trough groups in phase-locking $(0.19 \pm 0.04$ versus $0.28 \pm 0.05)$ or relative amplitude $(75 \% \pm 4$ percentage units versus $83 \% \pm 3$ percentage units, respectively) of the hippocampal fissure $\theta$-band responses to the tone presented alone prior to conditioning $\left(F_{(1,9)}=\right.$ alone. The hippocampal $\theta$-band responses were equally well phaselocked to the tone onset whether it was presented during $\theta$ (phaselocking value, mean \pm standard error of mean: $0.20 \pm 0.04$ ) or to a random brain state $\left(0.25 \pm 0.05\right.$; one-way ANOVA: $F_{(1,9)}=0.43$, $P=0.531$, group data not shown in figures). The amplitude of the $\theta$-band response to the tone was higher when it was delivered during $\theta$ ( $\theta$ ratio: $86 \% \pm 2$ percentage units) compared with when it was presented irrespective of neural state $(73 \% \pm 4$ percentage units; $\left.F_{(1,9)}=8.41, P=0.018\right)$. That is, $\theta$-contingent presentation elicited stronger $\theta$-band hippocampal responses to the tone.

Next, the effects of $\theta$ phase on hippocampal responses to the tone were examined in the five animals in which the tone was presented contingent on $\theta(\mathrm{T}+)$. We expected to see better phaselocked and possibly also bigger hippocampal $\theta$-band responses when tone onset overlapped with the fissure $\theta$ trough compared with when it started at $\theta$ peak. Equally strong $\theta$-band responses were elicited during the trough $(87 \% \pm 2$ percentage units) and the peak of the cycle $(85 \% \pm 2$ percentage units; paired samples $t$-test: $\left.t_{(4)}=1.74, P=0.156\right)$. That is, the relative amplitude of the $\theta$-band responses did not differ according to the phase of

$2.16, P=0.175$ and $F_{(1,9)}=2.30, P=0.163$, respectively). That is, animals in both groups responded similarly to the tone before it was used as a conditioned stimulus in trace eyeblink conditioning.

Previous studies indicate that the spontaneous level of hippocampal $\theta$ activity predicts subsequent learning (Berry and Thompson 1978; Nokia et al. 2009). Thus, to ensure there was no underlying difference in baseline $\theta$ between the Peak and the Trough groups, data from a 5-min stimulus-free recording conducted immediately before the first conditioning session were analyzed. Hippocampal $\theta$ ratios representing the relative power of $\theta$ in the Peak and Trough groups were comparable $(74 \% \pm 4$ percentage units versus $69 \% \pm 3$ percentage units, respectively; one-way ANOVA: $\left.F_{(1,9)}=1.03, P=0.337\right)$.

During subsequent conditioning, there were no differences between the groups in intertrial intervals (ITIs, repeated-measures [rm] ANOVA, main effect of group: $F_{(2,14)}=1.45, P=0.267$; interaction of group (3) and conditioning block $(5): F_{(8,56)}=0.67, P=$ 0.718). However, the ITI increased across the first five blocks (i.e., 10 sessions) of conditioning in all groups $\left(F_{(4,56)}=6.36, P<\right.$ $0.001)$. Mean ITI was $50.4 \pm 0.9 \mathrm{sec}$ during the first conditioning 
session and $57.6 \pm 1.7 \mathrm{sec}$ during the tenth conditioning session. This change was not part of the experimental design but it was expected in light of our previous observations indicating non- $\theta$ $(\mathrm{T}-$ ) periods occur more often as conditioning proceeds (Nokia and Wikgren 2014). In other words, periods of prominent spontaneous $\theta$ activity become less frequent across conditioning as the animal presumably becomes less attentive toward the, now familiar, situation. Further analyses indicated that no changes across training blocks or differences between groups in ITIs occurred during the six sessions (three blocks) of $\mathrm{T}-$ conditioning ( $\mathrm{rm}$ ANOVA: main effect of block: $F_{(2,26)}=2.11, P=0.142$, interaction of block and group: $F_{(4,26)}=0.16$, $P=0.955$ and main effect of group: $\left.F_{(2,13)}=0.52, \quad P=0.607\right)$. The average ITI during this latter training phase was $53.2 \pm 2.6 \mathrm{sec}$.

All in all, these analyses indicate no differences in baseline responding to the tone before it was used as the CS and no differences in either the level of spontaneous hippocampal $\theta$ activity or ITIs between the groups. This implies that any differences or changes across conditioning in hippocampal responding to the tone-CS or learning can be interpreted to be a result of our experimental manipulations. Note that as the tone elicited larger $\theta$-band responses in animals in which it was presented contingent on $\theta$ $(\mathrm{T}+$ versus random), this tone-alone treatment type was included as a covariate in the repeated-measures ANOVAs when examining differences between the experimental groups and changes across conditioning.

\section{Fissure $\theta$ trough-contingent conditioning enhanced hippocampal $\theta$-band responses to the CS while peak-contingent training impaired learning}

Our main aim in this experiment was to study whether the phase of $\theta$ oscilla-

tion at stimulus onset might have effects on learning about that stimulus. To this end, rabbits were trained for 10 sessions in trace eyeblink classical conditioning (see Materials and Methods), with the tone-CS timed to start either at the peak (Peak group) or the trough (Trough group) of the fissure $\theta$ cycle. The YC animals were trained simultaneously and received trials irrespective of their neural state. The phase-locking and relative amplitude of the hippocampal fissure $\theta$-band responses to the CS as a function of the trace eyeblink conditioning are presented in Figure $3 \mathrm{~A}-\mathrm{D}$.

Our hypothesis was that the hippocampal responses would be better phase-locked to the onset of the CS and possibly also higher in amplitude in the Trough group than in the Peak group. Both expectations were realized. First, phase-locking of hippo-
A

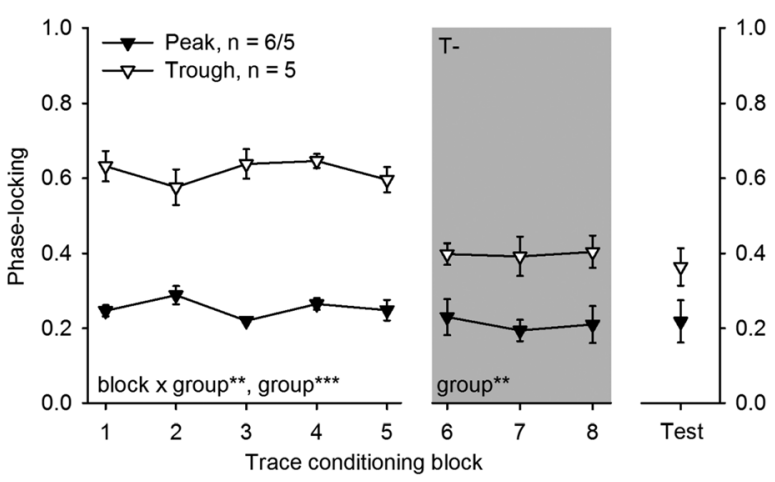

B

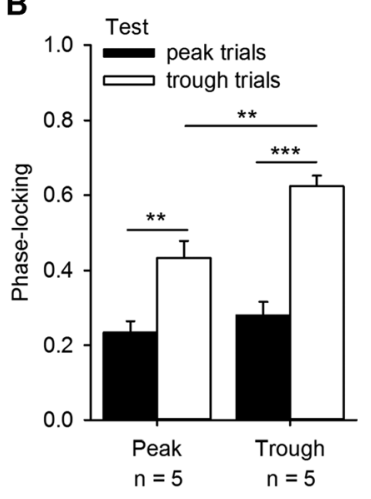

D

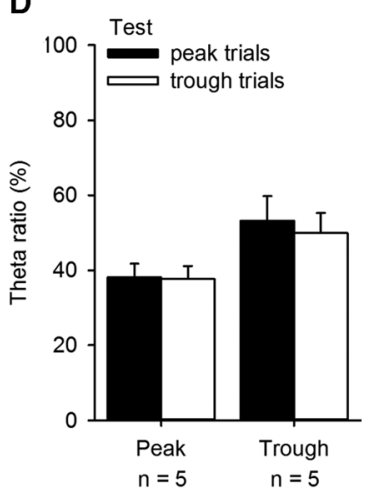

Figure 3. Trace eyeblink conditioning contingent on the phase of the hippocampal fissure $\theta$ cycle affects both the phase-locking $(A, B)$ and relative amplitude $(\theta$ ratio $\%, C, D)$ of hippocampal fissure $\theta$-band $(4-8 \mathrm{~Hz})$ responses to the conditioned stimulus (CS). (A) Hippocampal $\theta$-band responses to the CS were highly phase-locked if the CS was presented to the trough of the fissure $\theta$ cycle. In contrast, during the first 10 sessions of conditioning (i.e., the first five blocks). Note that during $\mathrm{T}-$ training (gray background) animals previously trained contingent on $\theta$ trough or peak were all trained contingent on the absence of $\theta$. Also note that in the Peak group $(n=6)$, one animal was dropped after the blocks of $\theta$ phase contingent conditioning due to breaking of the linear probe. Hence the number of animals in this group that proceeded to T- conditioning and the test phase is 5 . Asterisks refer to the results of repeated-measures (rm) ANOVA indicating an interaction of block and group cycle trough to well-trained animals (Test) resulted in higher phase-locking of fissure $\theta$-band responses. However, phase-locking remained higher in the group initially trained contingent on the $\theta$ trough (Trough group). Asterisks refer to results of paired samples $t$-tests (peak versus trough trials) and $\theta$-band responses ( $\theta$ ratio \%) to the CS was higher in the Trough than Peak group and consistently decreased across conditioning. Again, asterisks refer to repeated-measures ANOVA indicating significant

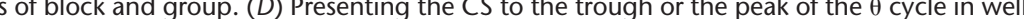
trained animals had no effect on the relative amplitude of the hippocampal $\theta$-band response. In all subtrained animals had no effect on the relative amplitud
plots vertical lines depict standard error of mean.

campal $\theta$-band responses to the CS was greater in the Trough group compared with the Peak group (rm ANOVA, main effect of block: $F_{(4,32)}=0.93, P=0.457$; interaction of block and tonealone treatment type: $F_{(4,32)}=0.93, P=0.460$; interaction of block and group: $F_{(4,32)}=4.31, P=0.007, \eta_{\mathrm{p}}^{2}=0.35$; main effect of tone-alone treatment type: $F_{(1,8)}=0.42, P=0.536$; main effect of group: $F_{(1,8)}=110.35, P<0.001, \eta_{\mathrm{p}}^{2}=0.93$ ) (see Fig. 3A, leftmost panel). Phase-locking remained stable across conditioning in both groups (Peak: $F_{(4,20)}=2.33, P=0.091$; Trough: $F_{(4,16)}=$ $2.84, P=0.059)$. Second, the relative amplitude of the hippocampal $\theta$-band responses to the CS was greater in the Trough group compared with the Peak group (main effect of group: $F_{(1,8)}=$ 11.46, $P=0.010, \eta_{\mathrm{p}}^{2}=0.59$ ) (see Fig. 3C, left-most panel). In addition, the relative amplitude of the responses decreased as a 
function of conditioning in both groups (main effect of block: $F_{(4,32)}=6.85, \quad P<0.001, \eta_{\mathrm{p}}^{2}=0.46$; interaction of block and group: $F_{(4,32)}=1.96, P=0.124$; interaction of tone-alone treatment type and block: $F_{(4,32)}=1.32, P=0.285$; main effect of tonealone treatment type: $\left.F_{(1,8)}=0.03, P=0.873\right)$.

Intrigued by the high level of phase-locking in the Trough group we also ran some analyses comparing our current data to results obtained in our previously published studies: In fact, the hippocampal responses elicited in the Trough group were more regular than those we have previously observed (Nokia and Wikgren 2014), either when the CS was presented contingent on hippocampal $\theta[\mathrm{T}+$, independent samples $t$-test on phaselocking of $\theta$-band responses to the CS elicited during the first conditioning block: $\left.t_{(14)}=9.93, P<0.001\right]$, contingent on the absence of $\theta\left[\mathrm{T}-, t_{(13)}=5.21, P<0.001\right]$ or irrespective of ongoing neural state [random, $\left.t_{(11)}=7.85, P<0.001\right]$. In contrast, the responses to the tone-CS presented at the $\theta$ peak were markedly less phase-locked and smaller in amplitude, and did not differ from those elicited in any of the groups in our earlier study (see [Nokia and Wikgren 2014], comparison to $\mathrm{T}+: t_{(15)}=1.30, P=$ 0.212 ; comparison to $\mathrm{T}-: t_{(14)}=0.25, P=0.804$; comparison to random: $\left.t_{(12)}=0.58, P=0.576\right)$.

In sum, as when presented alone, the tone presented as a CS during trace eyeblink conditioning elicited better phase-locked hippocampal $\theta$-band responses when it was timed to start at the trough of the spontaneously occurring $\theta$ oscillation than when it was timed to start at the $\theta$ peak (or irrespective of ongoing neural state, in the absence of $\theta$ or randomly during $\theta$, [Nokia and Wikgren 2014]). This effect was consistent across conditioning. In addition, the CS also elicited larger amplitude hippocampal $\theta$-band responses when it was presented to the trough compared with when it was presented at the peak. The amplitude of the $\theta$-band responses decreased across conditioning. This attenuation took place irrespective of the timing of the CS in relation to $\theta$ phase.

These results above suggest that $\theta$ phase-contingent conditioning might also affect learning at the behavioral level. Our hypothesis was that animals trained at the trough of hippocampal fissure $\theta$ oscillation would acquire trace eyeblink conditioning at the behavioral level faster/better than animals trained at the peak of oscillation or irrespective of their neural state. The behavioral results of the experiment are depicted in Figure 4A-C. Learned responding increased across the first five blocks of conditioning in all groups ( $\mathrm{rm}$ ANOVA, main effect of block: $F_{(4,52)}=$ 19.00, $P<0.001, \eta_{\mathrm{p}}^{2}=0.59$; interactions of block and tone-alone treatment type and block and group: $F_{(4,52)}=2.20, P=0.132$ and $F_{(8,52)}=0.98, P=0.436$, respectively; main effect of tone-alone treatment type: $F_{(1,13)}=1.68, P=0.218$ ) (see Fig. 4 A, left-most panel). However, the animals in the Peak group elicited significantly fewer conditioned responses (CRs) across the conditioning sessions compared with those in the $\mathrm{YC}$ and Trough groups (main effect of group: $F_{(2,13)}=5.33, P=0.020, \eta_{\mathrm{p}}^{2}=0.45$; pairwise Bonferroni-corrected comparisons: Peak versus Trough: $P=$ 0.053, Peak versus YC: $P=0.063$, Trough versus YC: $P=1.000$ ). Analysis of the cumulative percentage of CRs at the end of the first 10 sessions of conditioning confirmed this observation (one-way ANOVA: $F_{(2,14)}=6.08, P=0.013$; Bonferroni-corrected pairwise comparisons: Peak versus Trough: $P=0.096$, Peak versus YC: $P=0.014$, Trough versus YC: $P=1.000$ ) (Fig. $4 \mathrm{~B}$ ). The animals in the YC group made a conditioned response (CR) in $31 \%$ ( \pm 0.03 percentage units) of the trials compared with $27 \%$ $( \pm 0.02)$ in the Trough and only $16 \%( \pm 0.04)$ in the Peak group. Of the YC animals five out of six (83\%) reached the learning criterion of $60 \%$ CRs within the first 10 sessions of $\theta$-contingent conditioning. In contrast, in the Peak group only 1 out of $6(17 \%)$ and in the Trough group only two out of five (40\%) animals
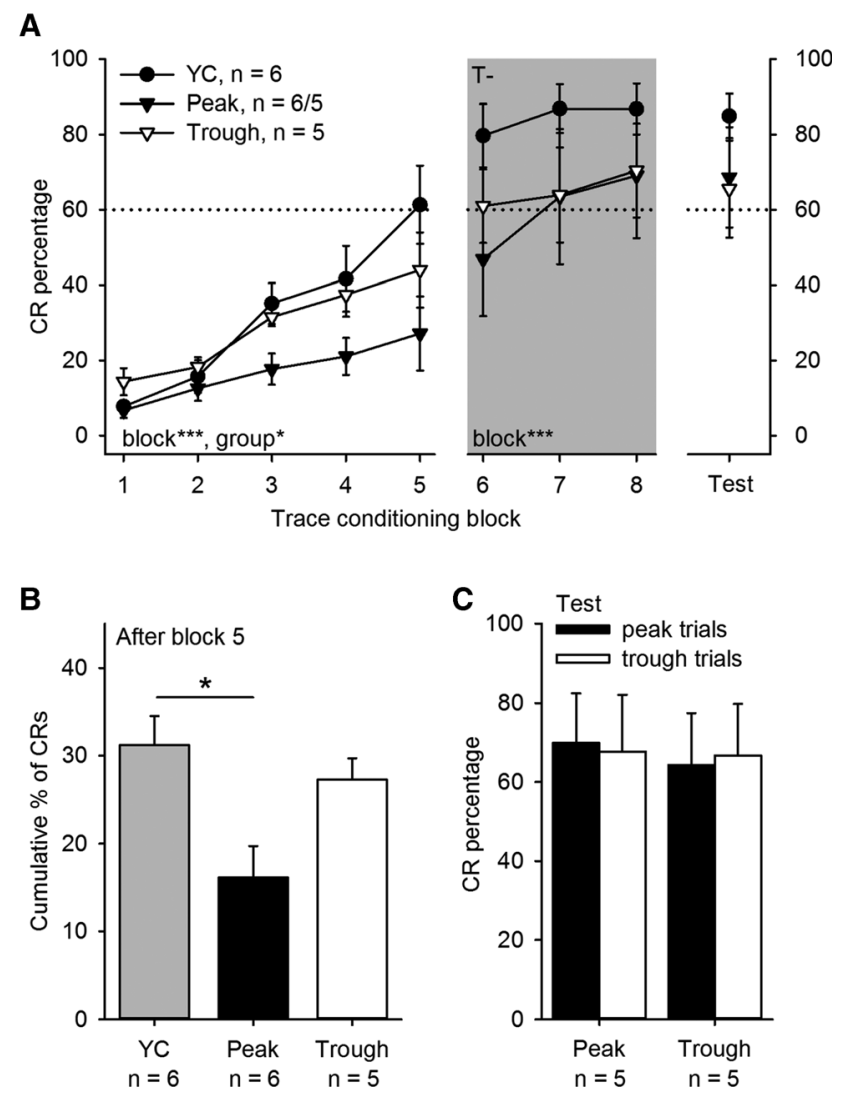

Figure 4. Trace eyeblink conditioning was retarded if the conditioning trials were consistently presented to the peak of hippocampal fissure $\theta$ $(A, B)$. In well-trained animals, presenting the conditioning trials contingent on hippocampal $\theta$ phase had no effect on conditioned responding (C). (A) Conditioned response (CR) percentage across the different phases of the experiment. Gray background indicates conditioning contingent on the absence of $\theta(\mathrm{T}-)$. Note that during $\mathrm{T}-$ training animals previously trained contingent on $\theta$ trough or peak were all trained contingent on the absence of $\theta$. For animals in the yoked control group, training was always random in relation to their neural state. Also note that in the Peak group $(n=6)$, one animal was dropped after the initial five blocks of $\theta$ phase contingent conditioning due to breaking of the linear probe. Hence the number of animals in this group that proceeded to T- conditioning and the test phase is 5. Asterisks refer to the results of repeated-measures ANOVA indicating that conditioned responding increased in all groups across the conditioning blocks $\left({ }^{* * *}\right) P<0.001$ and that there was a difference between groups during the first five conditioning blocks $P<0.05$. (B) The cumulative percentage of CRs in the Peak group was smaller than that in the YC group after the first five conditioning blocks. (C) In well-trained animals, conditioned responding was comparable whether the CS onset was timed to the trough or the peak of fissure $\theta$. Vertical lines depict standard error of mean.

reached criterion within the first 10 conditioning sessions. According to the binomial test, learning was significantly retarded in both the Peak and the Trough groups compared with YC: Peak: $P=0.001$; Trough: $P=0.035$. The YC animals performed the fifth CR after an average of $72 \pm 9$ paired trials whereas those in the Peak group needed $107 \pm 31$ trials and those in the Trough group $48 \pm 19$ trials to accomplish the same $\left(F_{(2,14)}=1.83, P=0.197\right)$.

To conclude, the acquisition of a behavioral learned response was retarded by $\theta$-contingent conditioning, when the CS was timed to start at the peak of the $\theta$ cycle. Despite enhancing hippocampal $\theta$-band responding to the CS, presenting conditioning trials to the trough of fissure $\theta$ had little effect on the acquisition of a behavioral CR. 


\section{Continued conditioning in the absence of $\theta(\mathrm{T}-)$ led to attenuated and less phase-locked hippocampal $\theta$-band responses while learned responding was further increased}

To maximize the number of animals that learned, we trained all the rabbits for another six sessions. Now, the animals previously trained contingent on $\theta$ phase (Peak and Trough groups) were trained in the explicit absence of $\theta(\mathrm{T}-)$ to maximize the number of animals that learned (Nokia and Wikgren 2014). The animals in the YC group continued to be trained irrespective of their neural state. In one animal initially assigned to the Peak group, the recording electrode broke during the night between sessions 13 and 14 and hence the animal was excluded from further training and analyses.

Our hypothesis was that $\mathrm{T}-$ conditioning would not change the hippocampal $\theta$-band responses to the CS considerably. If anything, we expected to see a decrease in phase-locking in the animals previously trained at the trough of $\theta$. First, possible changes in the $\theta$-band response amplitudes and phase-locking as a result of switching from $\theta$ phase-contingent to $\mathrm{T}$ - conditioning was studied in both groups (Peak and Trough) separately. Note that in Figure 3, data are presented compressed into blocks of two sessions; for this analysis, however, a paired-samples $t$-test was conducted on measures obtained during the last $\theta$ phasecontingent session (session 10) and the first $\mathrm{T}$ - session (session 11). In the Peak group, the phase-locking of the hippocampal fissure $\theta$-band responses to the CS remained the same, even when it was timed to start in the absence of $\theta\left[t_{(5)}=0.14, P=0.897\right]$, and the relative amplitude of the response decreased significantly $\left[t_{(5)}=7.01, P=0.001\right]$ (see Figs. 3A,C, respectively). In the Trough group, both the phase-locking as well as amplitude of the hippocampal $\theta$-band responses decreased significantly when the conditioning protocol was switched from $\theta$ trough-contingent to T $-\left[t_{(4)}=7.36, P=0.002\right.$ and $t_{(4)}=13.51, P<0.001$, respectively]. To summarize, switching from $\theta$ phase-contingent to T- conditioning attenuated hippocampal $\theta$-band responses to the CS. In addition, only in animals previously conditioned at $\theta$ trough, also the phase-locking of the responses to the CS decreased when training was switched to $\mathrm{T}-$.

Next, we studied whether hippocampal $\theta$-band responses changed across the $\mathrm{T}-$ conditioning (three blocks of two sessions) and whether the differences between groups in hippocampal responding (Peak versus Trough) were still evident even though conditioning was now carried out in an identical way, that is, in the explicit absence of $\theta$. The CS continued to elicit better phaselocked responses in the animals previously trained at $\theta$ trough compared with those previously trained at $\theta$ peak also during the T- conditioning ( $\mathrm{rm}$ ANOVA, main effect of group: $F_{(1,7)}=$ 15.35, $P=0.006, \eta_{\mathrm{p}}^{2}=0.69$; main effect of block: $F_{(2,14)}=0.12$, $P=0.884$; interaction of block and tone-alone treatment type: $F_{(2,14)}=1.67, P=0.223$; interaction of block and group: $F_{(2,14)}=$ $0.74, P=0.494$; main effect of tone-alone treatment: $F_{(1,7)}=$ 2.64, $P=0.148$ ) (see Fig. 3A middle panel, gray background). However, there were no significant differences between groups or changes across the $\mathrm{T}$ - conditioning in the relative amplitude of the $\theta$-band responses to the CS (rm ANOVA, main effect of group: $F_{(1,7)}=4.30, P=0.077$, main effect of block: $F_{(2,14)}=$ 1.69, $P=0.220$; interaction of block and tone-alone treatment type: $F_{(2,14)}=0.22, P=0.805$; interaction of block and group: $F_{(2,14)}=2.59, P=0.110$; main effect of tone-alone treatment: $F_{(1,7)}=0.57, P=0.474$ ) (see Fig. 3C middle panel, gray background). To summarize, during $\mathrm{T}-$ conditioning, phase-locking of hippocampal $\theta$-band responses to the CS diminished yet continued to be higher in animals previously trained at $\theta$ trough compared with those previously trained at $\theta$ peak. Hippocampal $\theta$-band response amplitude was diminished overall compared with $\theta$ phase-contingent conditioning and a statistically significant difference between the previous Peak and Trough groups was no longer evident.

In terms of learning trace eyeblink conditioning, we expected animals in the experimental groups (Peak and Trough) to learn the task during the $\mathrm{T}$ - training, if they had not already done so during the first 10 conditioning sessions conducted contingent on $\theta$. We used $\mathrm{T}-$ training because in our previous study (Nokia and Wikgren 2014) it led to a greater proportion of animals learning compared with random presentation of training trials (note that $\mathrm{T}+$ conditioning had no such effect). Conditioned responding continued to increase in all groups during the second phase of training (rm ANOVA: main effect of block $(3): F_{(2,24)}=12.71, P=$ $0.001, \eta_{\mathrm{p}}^{2}=0.51$; interaction of group (3) and block: $F_{(2,24)}=$ $3.09, P=0.062$; interaction of tone-alone treatment type and block: $F_{(2,24)}=0.49, P=0.544$; main effect of group: $F_{(2,12)}=$ $0.44, P=0.655$; main effect of tone-alone treatment type: $F_{(1,12)}=1.85, P=0.199$ ) (see Fig. $4 \mathrm{~A}$ middle panel, gray background). At the end of the $\mathrm{T}-$ conditioning, the cumulative percentage of CRs was $50 \%$ in the YC group compared with $32 \%$ in the Peak and $40 \%$ in the Trough group $\left(F_{(2,13)}=2.72, P=\right.$ $0.103)$. By the end of $16 \mathrm{~d}$ of conditioning, all (100\%) of the YC animals, three out of five (60\%) animals previously trained at $\theta$ peak and 4 out of $5(80 \%)$ animals previously trained at $\theta$ trough reached the learning criterion of $60 \%$ CRs during at least one session. The binomial test could not be run because all the animals in the YC group learned. Of the animals that eventually learned, those in the YC group reached the traditional learning criterion of eight CRs in nine consecutive trials after an average of $401 \pm$ 43 trials whereas those first trained at $\theta$ peak and then in the absence of $\theta$ required $547 \pm 54$ trials and those first trained at $\theta$ trough and then in the absence of $\theta 456 \pm 71$ trials to perform at the same level $\left(F_{(2,10)}=1.62, P=0.246\right)$. To conclude, after $\mathrm{T}-$ conditioning the learning outcome in the Peak and Trough groups was similar to that of the YC group.

\section{High-amplitude, well phase-locked hippocampal fissure $\theta$-band responses to the conditioned stimulus early in training predicted better learning of trace eyeblink conditioning}

Previous studies indicate a correlation between hippocampal function and learning only, or at least especially, early in training (Nokia et al. 2009). To study the link between hippocampal $\theta$ band responses and learning, we calculated correlations between the relative amplitude/phase-locking of hippocampal $\theta$-band responses to the CS during the first conditioning session and the percentage of CRs elicited during conditioning (blocks 1-8). For this, animals from both the Peak and the Trough groups were pooled. A high $\theta$ ratio in response to the CS during the first conditioning session predicted a higher CR percentage during conditioning blocks $3-6(r=0.656-0.694, P=0.018-0.028, n=11)$. The correlations between the $\theta$ ratio and conditioned responding during blocks $1-2$ and 7-8 were not statistically significant $(r=$ $0.332-0.495, P=0.146-0.318, n=11 / 10)$. In addition, highly phase-locked responding during the very first conditioning session predicted a higher percentage of CRs during conditioning blocks 3 and $4(r=0.760 / 0.693, P=0.007 / 0.018, n=11$, respectively). There was also a close to significant correlation with conditioned responding during block $5(r=0.553, P=0.078, n=11)$. Correlations with CR percentage during blocks 1,2 , and $6-8$ were not significant $(r=0.137-0.466, P=0.149-0.706, n=11)$. To summarize, high-amplitude, temporally uniform hippocampal $\theta$-band responses to the CS at the start of conditioning anticipated good learning. Hippocampal responding early in training was 
correlated to conditioned responding specifically in the middle of training, when the conditioned response is being acquired and its performance rate starts to rise rapidly (Prokasy 1984).

\section{$\theta$ phase at CS onset did not affect conditioned responding in well-trained animals}

After 16 sessions of conditioning, two more sessions were carried out, now reverting back to the procedure of presenting the trials contingent on hippocampal $\theta$ phase for animals in the Peak and Trough groups. However, now half the trials were presented to the trough and half to the peak of the $\theta$ cycle recorded from the hippocampal fissure, in a random order. Animals in the YC group remained to be conditioned irrespective of their neural state. Our hypothesis was that a conditioned response would be more likely to occur in response to a CS presented at $\theta$ peak compared with when it was presented at $\theta$ trough. This would have indicated superior memory retrieval at $\theta$ peak (Hasselmo et al. 2002).

The CS continued to elicit better phase locked $\theta$-band responses from the hippocampal fissure if it was timed to start at the trough of the $\theta$ cycle compared with the peak of the $\theta$ cycle (rm ANOVA with group [Peak versus Trough] as a between-subjects factor: main effect of trial type [peak versus trough]: $F_{(1,8)}=$ 109.83, $\left.P=0.001, \eta_{\mathrm{p}}^{2}=0.93\right)$. The analysis also revealed a significant interaction of trial type and group $\left(F_{(1,8)}=7.83, P=\right.$ $\left.0.023, \eta_{\mathrm{p}}^{2}=0.50\right)$ and a main effect of group $\left(F_{(1,8)}=7.43, P=\right.$ $0.026, \eta_{p}^{2}=0.48$ ) (see Fig. 3B). Due to the interaction, groups were further analyzed separately using paired samples $t$-test. This confirmed that the CS elicited better phase-locked $\theta$-band responses in both groups when presented to the trough of $\theta$ during the test sessions (Peak: $t_{(4)}=4.93, P=0.008$; Trough: $t_{(4)}=10.58$, $P<0.001$; see Fig. 3B). However, the CS also still elicited better phase-locked responses in animals initially trained at $\theta$ trough compared with animals initially trained at $\theta$ peak, when it was presented to the trough of the $\theta$ cycle (one-way ANOVA: $F_{(1,8)}=$ $12.98, P=0.007)$. The phase-locking of responses to the CS was similar in both groups when it was timed to commence at the peak of the fissure $\theta$ cycle $\left(F_{(1,8)}=0.93, P=0.363\right)$. Also, there were no statistically significant differences between groups (Peak versus Trough group, trials presented at the peak/trough: $F_{(1,8)}=$ $3.94 / 3.75, P=0.083 / 0.089$ ) or effects of $\theta$ phase (trials presented to peak versus trough, Peak group: $t_{(4)}=0.06, P=0.578$; trials presented to peak versus trough, Trough group: $t_{(4)}=1.95, P=0.123$ ) on the relative amplitude of the hippocampal fissure $\theta$-band responses (see Fig. 3D). However, the relative amplitude of the $\theta$-band responses elicited in response to the CS in the hippocampal fissure attenuated in animals initially trained at the peak of $\theta$ when training was switched back from $\mathrm{T}-$ to $\theta$ phase-contingent (last $\mathrm{T}$ - session versus first test session, paired samples $t$-test: $t_{(4)}=$ $5.44, P=0.006)$. The same was not true for the Trough group $\left(t_{(4)}=1.96, P=0.122\right)$. Overall, conditioned responding during the two test sessions remained at the same level compared with the last $\mathrm{T}-$ conditioning block (paired samples $t$-tests: YC: $t_{(5)}=$ 0.81, $P=0.457$; Peak: $t_{(4)}=0.01, P=0.990$; Trough: $t_{(4)}=2.09$, $P=0.105$; see Fig. 4A, right-most panel). Last but most important, behavioral responding did not differ between the peak and trough trials (paired samples $t$-tests: Peak group: $t_{(4)}=0.48, P=0.654$; Trough group: $t_{(4)}=0.69, P=0.526$; see Fig. $4 \mathrm{C}$ ).

To summarize, $\theta$ phase continued to modulate hippocampal responses to the CS even after extensive training. In addition, animals initially trained at $\theta$ trough continued to exhibit better phase-locked hippocampal $\theta$-band responses to the CS compared with animals initially trained at $\theta$ peak. This effect was limited to trials during which the CS commenced at $\theta$ trough. Most important, despite these clear effects on hippocampal responding, $\theta$ phase had no effect on memory retrieval at the behavioral level.

\section{Discussion}

Hasselmo and colleagues (Hasselmo et al. 2002; Hasselmo and Stern 2014) have proposed that the function of hippocampal $\theta$ oscillations in learning and memory is to enable separate time windows for the encoding and retrieval of memories by regulating information flow within the hippocampo-entorhinal loop. Inspired by this idea, we studied whether the phase of hippocampal $\theta$ oscillation at stimulus onset has an effect on hippocampal responding to that stimulus and whether this affects learning about that stimulus in the context of classical eyeblink conditioning in rabbits. Our results indicate that the most regular hippocampal responses occurred to stimuli presented at the trough of fissure $\theta$, whereas the responses to stimuli presented at the peak of fissure $\theta$ were less organized. Overall, high-amplitude, temporally regular hippocampal $\theta$-band responses early in training predicted better learning. Presenting the CS at $\theta$ peak retarded learning whereas the acquisition of a learned response was little affected in the Trough group, despite the enhanced hippocampal $\theta$-band responses to the CS. Across continued conditioning and in well-trained animals, the association between hippocampal responses to the CS and behavioral learned responses vanished, suggesting that the role for hippocampal function in learning is time-limited. In sum, $\theta$ phase predetermines both hippocampal responding to peripheral stimuli and learning about those stimuli indicating that it is involved in regulating processing of information in the brain up to a behaviorally relevant degree.

According to the computational model by Hasselmo and colleagues (Hasselmo et al. 2002; Hasselmo and Stern 2014), the phase of hippocampal $\theta$ oscillation determines whether the hippocampus preferentially processes input from the entorhinal cortex, leading to memory encoding, or from the CA3 autoassociative network, leading to memory retrieval. To a certain degree, our results comply with the model: a brief auditory stimulus evoked large and temporally well-organized responses at the $\theta$-band within the hippocampus when timed to start specifically at the trough of a $\theta$ cycle recorded from the hippocampal fissure. In fact, the hippocampal responses elicited were more regular than those we have previously observed (Nokia and Wikgren 2014), either when the CS was presented contingent on hippocampal $\theta$ or irrespective of ongoing neural state. In contrast, the responses to the tone-CS presented at the $\theta$ peak were markedly less phase-locked and smaller in amplitude, and did not differ from those elicited in any of the groups in our earlier study (Nokia and Wikgren 2014). The high phase-locking of $\theta$-band responses in the Trough group resembles the $\theta$ reset observed in the dentate gyri of rats performing a working memory task involving both visual and auditory peripheral stimuli (Givens 1996). It has been demonstrated in rats that LTP is preferentially induced if stimulation is timed to occur during the peak of the CA1 $\theta$ (trough of fissure $\theta$ ) not only when the oscillation occurs spontaneously (Holscher et al. 1997) but also when it is evoked and reset by a peripheral stimulus (McCartney et al. 2004). Thus, phase-locking or resetting of hippocampal $\theta$ oscillation to the stimulus onset creates windows of opportunity for enhanced processing of subsequent relevant stimuli. Together with these earlier findings our current results imply that hippocampal processing of related peripheral stimuli occurring close in time (within $500 \mathrm{msec}$ to $1 \mathrm{sec}$ of each other) could be brought to or near the maximum by presenting the stimuli consistently at the hippocampal fissure $\theta$ trough. In further studies, conditioning should be conducted in a manner where both the CS and the unconditioned stimulus are presented consistently to the trough/ peak of the hippocampal $\theta$ oscillation. One would expect superior learning if both stimuli occur at fissure $\theta$ trough.

Consistent with the theory by Hasselmo et al. (2002), compared with a yoked control group, learning was severely retarded 
when the conditioned stimulus was presented at the peak of fissure $\theta$. Learning was also not improved in the group trained at the fissure $\theta$ trough. That is, even though the conditioned stimulus evoked unusually regular responding in the hippocampus, and even though high regularity, once again (Nokia and Wikgren 2014), predicted good learning in general, it was not enough to improve the acquisition of the behavioral learned response. This suggests the existence of a ceiling effect where hippocampal responding seems to support learning to a limited degree only, no matter how regular or efficient it is. In fact, in healthy young animals learning seems to be most efficient when the to-be learned associations are presented randomly or in the absence of hippocampal $\theta$ and with a long enough ITI (Nokia and Wikgren 2014). Our current and earlier results are at odds with some previous findings on enhanced learning and neural responses following $\theta$-contingent trace eyeblink conditioning in young healthy animals (Griffin et al. 2004; Darling et al. 2011). These discrepancies in results might be explained by differences in, for example, the algorithm for detecting $\theta$ or the duration of the CS which here was $200 \mathrm{msec}$ in comparison to $100 \mathrm{msec}$ used in the studies cited above. It might be that the longer duration of the CS used here made its presentation less specific to a certain phase of the $\theta$ oscillation as the duration of a single full cycle of a $6 \mathrm{~Hz}$ oscillation is $<200 \mathrm{msec}$.

In addition to the effects of $\theta$ phase on the encoding of incoming information we also studied its effects on the retrieval of already established memories. According to the theory of Hasselmo and colleagues $(2002,2014)$, during the peak of the $\theta$ cycle recorded near the hippocampal fissure, entorhinal input is suppressed and the CA3 autoassociative network activated, which supports the retrieval of already encoded information. However, in the present study, there was no effect of $\theta$ phase on memory retrieval in well-learned animals. Although hippocampal responses to the CS were, once again, temporally well-organized when presented to the trough of the $\theta$ cycle and less organized when presented to the peak, conditioned responding at the behavioral level did not differ according to the timing of the CS relative to the fissure $\theta$ phase. Thus, efficient hippocampal processing seems to be crucial only in the very early stage of learning, when the CSUS association is first realized and CRs start to emerge at an increasing rate (Prokasy 1984). It would seem that the hippocampus has only a modulatory, if any, role in the late stages of CR acquisition or in the retention of the CR, leaving other brain structures such as the prefrontal cortex (Weiss and Disterhoft 2011) and/or the cerebellum (Gould and Steinmetz 1996) in charge. To better pinpoint the timing of hippocampal dependence of learning, the effects of $\theta$ phase-contingent stimulus presentation on memory retrieval should be tested earlier in the conditioning process, ideally at several different intervals from the onset of conditioning, in subjects otherwise trained irrespective of their neural state. It is also noteworthy that the theory by Hasselmo and colleagues $(2002,2014)$ was developed to explain mechanisms of memory retrieval and encoding during episodic memory modeled by spatial navigation tasks in rodents. Although also a form of episodic learning, eyeblink classical conditioning in rabbits might be governed by slightly different rules, which might explain the contradictions between our current results and the theory by Hasselmo and colleagues (2002); Hasselmo and Stern (2014).

In conclusion, to our knowledge, this is the first study to demonstrate that the timing of a peripheral stimulus to a certain phase of hippocampal $\theta$ oscillation produces robust effects on the synchronization of neural responses and affects learning at the behavioral level. That is, the phase of the hippocampal $\theta$ oscillation seems to be a means of regulating the processing of information in the brain up to a behaviorally relevant degree. Our results also support the notion of a time- and otherwise limited role for hippocampal processing in associative learning: highamplitude, phase-locked hippocampal responses to the CS seem crucial only for the initial stage of classical conditioning, i.e., associating the CS with the unconditioned stimulus, and enhancing these responses does not automatically lead to better learning.

\section{Materials and Methods}

\section{Subjects}

The subjects were 17 adult female New Zealand White rabbits (Lidköpings kaninfarm, Sweden) weighing $\sim 3.3 \mathrm{~kg}$ at the time of surgery. The rabbits were housed in individual cages on the premises of the animal research unit of the University of Jyväskylä. Food and water were freely available, and room temperature and humidity were controlled. The rabbits were maintained on a 12/ $12 \mathrm{~h}$ light-dark cycle, with lights on at $8.00 \mathrm{a} . \mathrm{m}$. All procedures were conducted during the light portion of the cycle. All the experimental procedures were implemented in accordance with Directive 2010/63/EU of the European Parliament and of the Council on the care and use of animals for research purposes.

\section{Surgery}

Subcutaneous injections of an analgesic solution $(0.1 \mathrm{~mL}$ of 0.3 $\mathrm{mg} / \mathrm{mL}$ buprenorphine [Temgesic, Schering-Plough Europe] diluted in $0.9 \mathrm{~mL}$ of $0.9 \% \mathrm{NaCl}$, dose: $2 \mathrm{~mL}$ ) and of an antiinflammatory drug $(50 \mathrm{mg} / \mathrm{mL}$ carprofen [Rimadyl vet, Pfizer Inc. Animal Health], dose: $0.1 \mathrm{~mL} / \mathrm{kg}$ ) were administered $>30$ min prior to surgery. The rabbits were anesthetized with an i.m. injection of ketamine-xylazine cocktail $(7.8 \mathrm{~mL}$ of $50 \mathrm{mg} / \mathrm{mL}$ Ketaminol vet [Intervet International B.V.] mixed with $2.8 \mathrm{~mL}$ of $20 \mathrm{mg} / \mathrm{mL}$ Narcoxyl vet [Intervet International B.V.]). A dose of $0.8 \mathrm{~mL} / \mathrm{kg}$ of the cocktail was injected i.m. before surgery. During surgery, additional doses of either the cocktail or ketamine alone were injected subcutaneously approximately every 20-30 min or as needed. At the beginning of surgery, the rabbit was placed in a stereotaxic instrument (Kopf Instruments) with the bregma positioned $1.5 \mathrm{~mm}$ higher than the lambda. Eye gel was administered to prevent the animal's eyes from drying. A longitudinal incision was made to the scalp and four stainless-steel anchoring screws $(5 \mathrm{~mm}$ anterior and $5 \mathrm{~mm}$ lateral to the bregma; $13 \mathrm{~mm}$ posterior and $5 \mathrm{~mm}$ lateral to the bregma) were attached to the skull. The screws were connected together and served as a reference for the electrophysiological recordings. Next, a piece of skull was removed above the left hippocampus and the dura cut to expose the surface of the brain. A 32-channel linear probe (A1 $\times 32-10$ mm-100-177, H32 package, NeuroNexus) attached to a microdrive (nDrive xL, NeuroNexus) was chronically implanted into the dorsal hippocampus $5 \mathrm{~mm}$ posterior, $5.5 \mathrm{~mm}$ lateral, and $\sim 4 \mathrm{~mm}$ below the surface of the brain, aiming the tip of the probe at the dentate gyrus. The opening in the skull was sealed using Kwik-Sil (World Precision Instruments) silicone. Last, the probe and microdrive were cemented in place with dental acrylic. For three animals, instead of the linear probe, six monopolar recording electrodes made of Teflon-insulated stainless steel wire (bare diameter $125 \mu \mathrm{m}$ ) mounted inside 27-gauge hypodermic stainless steel tubing were chronically implanted into the left dorsal hippocampus 5-6 $\mathrm{mm}$ posterior, $4-7 \mathrm{~mm}$ lateral, and $6.5-7.4$ $\mathrm{mm}$ below the bregma, aiming at the hippocampal fissure and dentate gyrus. The data from these animals were combined with the data from the animals implanted with linear probes. Metoclopramide (dose $0.1 \mathrm{~mL} / \mathrm{kg}$, concentration $5 \mathrm{mg} / \mathrm{mL}$; Primperan [Sanofi Winthrop Industrie]) was injected subcutaneously immediately after surgery to facilitate normal feeding and drinking. Analgesic (buprenorphine, see above for details) was administered every $8 \mathrm{~h}$ for the next $24-48 \mathrm{~h}$ depending on the recovery rate of the animal.

\section{Conditioning procedure}

During the recordings, the rabbits were placed in a Plexiglas restraining box allowing free movement of the head and located 
in a ventilated, electrically insulated, and sound-attenuated conditioning chamber. A fan located inside the conditioning chamber behind the rabbit created a steady background noise of $\sim 65$ $\mathrm{dB}$. At least $1 \mathrm{wk}$ was allowed for post-surgical recovery before commencing the experiment. During the recovery period, the animals were habituated to the recording chamber and neural signals recorded briefly (10-20 min) to adjust the position of the linear probe, using the microdrive. The probe was positioned so that $\mathrm{CA} 1$ ripples, $\theta$ and $\mathrm{CA} 3$ ripples were visible.

LabView (National Instruments) and E-Prime software (Psychology Software Tools Inc.) were used to control the presentation of the stimuli. Briefly, the signal from one electrode site near the hippocampal fissure was monitored online in 1-s sweeps refreshed every $50 \mathrm{msec}$. For each sweep, the Fast Fourier Transform was calculated with a resolution of $1 \mathrm{~Hz}$. The $\theta$ ratio (\%) was calculated as the ratio between the power of the signal at $5-8 \mathrm{~Hz}$ divided by the power of the signal at $1-12 \mathrm{~Hz}$.

\section{Tone-alone session: testing response modulation according to $\theta$ phase}

To study response modulation as a function of hippocampal $\theta$ phase, before conditioning, an $80-\mathrm{dB}, 4-\mathrm{kHz}, 200-\mathrm{msec}$ tone later to be used as the CS was presented 300 times (minimum intertrial interval (ITI) $10 \mathrm{~s}$ ). For five animals later assigned to the experimental groups, the tone was presented during periods of dominant $\theta(\mathrm{T}+)$ recorded from the fissure. Stimuli were delivered when the $\theta$ ratio exceeded $80 \%$. For six animals later assigned to the experimental groups, tones were presented irrespective of their brain state, using the ITIs from the first five animals (to which the tone was presented contingent on a high $\theta$ ratio). For the animals assigned to the yoked control group, stimuli were presented irrespective of their brain state. The CS-alone treatment type $(T+$ versus random) was included as a covariate in the repeated-measures ANOVAs when examining differences between the experimental groups and changes across training.

\section{$\theta$ phase-contingent trace eyeblink conditioning (Peak/Trough)}

During conditioning, the CS, a 80- $\mathrm{dB}, 4-\mathrm{kHz}, 200-\mathrm{msec}$ tone, was paired with the unconditioned stimulus (US), a 100-msec air puff toward the eye. A 500-msec trace period was used, creating a 700-msec onset-to-onset interval. Sixty trials per session were conducted with a minimum ITI of $35 \mathrm{~s}$. For one group of animals, the CS was timed to commence at the trough of hippocampal fissure $\theta$ (Trough, $n=5$ ) and for the other group it was timed to start at the $\theta$ peak (Peak, $n=6$ ). Examples of the timing of the conditioning stimuli relative to hippocampal $\theta$ are presented in Figure 1B. Grouping was random. Using LabView, the CS was timed to commence at a certain phase of the $\theta$ cycle by using a combination of the $\theta$ ratio $(>80 \%)$ and simple threshold triggering: the signal had to pass through two threshold values either on its way up (rising) or on its way down (falling). Although the algorithm was very simple, and by no means perfect, the setup worked well. The instantaneous phase of the $\theta$ cycle at CS onset was -0.03 radians (SEM 0.05) in the Peak group and 3.01 (SEM 0.05) radians in the Trough group during the first conditioning session, indicating very good accuracy in timing. (Note that for the trough group, negative phase values were corrected $(x+2 \pi)$ so that the distribution peak was set at around $\pi$.) A group of yoked control (YC, $n=$ $6)$ animals were trained together with animals in the Peak $(n=3)$ and Trough $(n=3)$ groups, that is, the YC group received conditioning trials irrespective of their neural state. Animals were assigned to the YC group on the basis of broken electrodes (no signal). Ten conditioning sessions were carried out on consecutive days.

\section{Trace eyeblink conditioning contingent on the absence of $\theta(T-)$}

After the initial training period, conditioning was continued for another six daily sessions, but now trials were only triggered when the animal in the experimental group (Peak/Trough) exhibited low $\theta$ power $(\mathrm{T}-, \theta$ ratio $<35 \%)$.

\section{Testing the effect of $\theta$ phase on memory and learned performance}

Finally, the effects of fissure $\theta$ phase on retrieval of the memory trace/performance of the conditioned response (CR) (Hasselmo et al. 2002; Hasselmo and Stern 2014) were tested. Animals were presented with 60 paired trials, of which 30 were timed to commence at the peak and 30 at the trough of fissure $\theta$, in a random order. Another test session was performed the next day to accumulate 60 trials delivered to the peak and 60 trials delivered to the trough of $\theta$.

\section{Recordings and data analysis}

Bipolar electromyogram (EMG) was recorded using stainless steel wire hooks placed around the right upper and lower eyelids for the duration of the training session. To acquire neural measures, a low-noise preamplifier (MPA8I or MPA32, MultiChannel Systems (MCS)) was directly attached to the electrode coupler anchored with dental acrylic to the rabbit's head. A flexible, insulated cable was used to connect the animal to the filter amplifier (FA64I, filter: 1-5000 Hz, MCS). All signals were digitized (USBME-64 System, MCS) and recorded with Mc_Rack software (MCS), using a 20-kHz sampling rate. Finally, all signals were digitally band-pass filtered between 1 and $500 \mathrm{~Hz}$ (high-pass: RC, lowpass: fourth-order Bessel) and stored using a 2-kHz sampling rate. MATLAB (The MathWorks Inc.), and SPSS (SPSS Inc.) were used for offline data analysis.

\section{Eyeblinks}

The EMG signal was high-pass filtered off-line $(>100 \mathrm{~Hz})$ and Hilbert-transformed. An envelope curve following the peaks of the signal was calculated. Baseline EMG activity was defined for each animal and session as the mean of the peak EMG amplitude during a 500-msec pre-CS period (MEANpre). Also determined was the mean of the standard deviation of the EMG activity during the 500-msec pre-CS period (SDpre). Eyeblinks were defined as EMG activity exceeding a threshold of [MEANpre $+4 \times$ SDpre] for at least $10 \mathrm{msec}$. Trials with eyeblinks during the $100-\mathrm{msec}$ period immediately preceding CS onset were rejected. Eyeblinks during the latter half (250-msec period) of the 500 -msec trace period were counted as conditioned responses. As in our previous studies (Nokia and Wikgren 2014) on trace eyeblink conditioning, the learning criterion were considered to be met when the subject performed $\geq 60 \%$ CRs during at least one conditioning session. Learning rate was quantified as the cumulative percentage of CRs, the number of trials to the fifth CR and the number of trials to meet the criterion of eight CRs on nine consecutive trials, which has been used to indicate asymptotic learning (see, for example, Berry and Thompson 1978).

\section{Relative magnitude of hippocampal $\theta$-band $(4-8 \mathrm{~Hz})$ oscillations: $\theta$ ratio}

$\theta$ ratio (Nokia et al. 2009) was obtained from a 5-min baseline recording conducted immediately prior to the first conditioning session. To this end, the hippocampal fissure signal was analyzed in 1500-msec sweeps using the FFT. From the FFT result, the ratio (\%) of the power of the signal at the $\theta$ band $(4-8 \mathrm{~Hz})$ compared with that at the $\theta$ and delta bands $(1-8 \mathrm{~Hz})$ was calculated. To study the relative amplitude of the $\theta$-band responses to the conditioning stimuli, the hippocampal $\theta$ ratio was calculated from 700-msec period immediately following CS onset. For statistical analyses, the $\theta$ ratio during the CS and subsequent trace period (700 $\mathrm{msec}$ ) was averaged over sessions (correlation with learning) and then over blocks of two sessions (changes across training and differences between groups).

\section{Phase-locking of hippocampal $\theta$-band $(4-8 \mathrm{~Hz}$ ) responses}

To assess the temporal accuracy of the $\theta$-band responses to the conditioning stimuli, a phase-locking value (PLV) (Palva et al. 
2005) was calculated. The PLV is based on amplitude-normalized phase information and is thus resistant to changes or differences in signal amplitude. This allows comparable measures to be obtained from data recorded over time in multiple subjects. The hippocampal LFP data were first band-pass filtered between 4 and 8 $\mathrm{Hz}$. Then, a Hilbert transform was run on the signal to obtain the phase information, and the amplitude of the signal normalized to 1 by dividing each data point by its absolute value. Finally, the PLV was obtained by averaging over 60 trials (one session) and taking the absolute value of the mean. The PLV varies between 0 and 1, 0 indicating no phase locking and 1 indicating perfect phase locking. For statistical analyses, the mean of the PLV during the CS and subsequent trace-period (700 msec) was derived and averaged over blocks of two sessions. Note that for the analysis of the CS-alone data in the animals that received the CS contingent on $\theta$ (T+, impact of peak versus trough), 60 trials during which the CS onset was closest to the peak and 60 trials during which it was closest to the trough of the cycle were selected and PLVs calculated for these. This ensured comparability of the analyses in relation to those conducted on the data obtained during conditioning. For the comparisons between the CS-alone treatment types (T+ versus random) and those between the experimental groups-to-be (Peak versus Trough), all $300 \mathrm{CS}$-alone trials were used for calculating the PLV.

\section{Current source density}

To visualize the sources and sinks of LFPs in different hippocampal cell layers, current source density analysis (for the theoretical basis see Nicholson and Freeman [1975] and Mitzdorf [1985]) was performed on data recorded during the tone-alone session in selected animals. The result for one representative animal was visualized with MATLAB (Fig. 2) and corresponds to the unscaled second derivative of the potential as a function of recording depth (see also Bragin et al. 1995).

\section{Statistical analyses}

Repeated-measures analysis of variance (ANOVA), with training blocks of two sessions as a within-subjects factor and group as a between-subject factor, was used to analyze changes across training. Type of CS-alone treatment (random versus T+) was included as a two-level covariate $(0 / 1)$. Whenever a significant interaction of block and group emerged, separate repeated-measures ANOVAs were conducted for each group, using blocks as a within-subjects factor. Greenhouse-Geisser corrected $P$-values are reported when the sphericity assumption was violated according to Mauchly's test. One-way ANOVA was used in group comparisons when single variables were tested. Bonferroni corrected $P$-values were used for post hoc comparisons. Binomial test was used to compare the proportions (\%) of animals that learned in each group. Pearson's correlation coefficient was used to analyze the connection between hippocampal $\theta$-band responses to the CS and learning. Finally, paired samples $t$-tests were used to analyze differences within subjects.

\section{Histology}

Rabbits were anesthetized with an i.m. injection of ketaminexylazine cocktail and then overdosed with an i.v. injection of pentobarbital (Mebunat vet, Orion-Yhtymä Oyj). Next, the brain was perfused with physiological saline followed by $9 \%$ formalin solution through the ascending aorta. The locations of the electrode tips were marked by passing a DC current $(200 \mu \mathrm{A}, 5 \mathrm{sec})$ through them. The brain was then removed and stored in formalin for several days. The brain was coronally sectioned with a vibratome into $60-\mu \mathrm{m}$-thick slices. The slices were attached to gelatinized slides, dried, and stained with Prussian blue and cresyl violet. The electrode locations were determined with the help of a microscope.

\section{Acknowledgments}

We thank Lauri Viljanto for technical help. The work was supported by the Academy of Finland (grant no. 139767 to M.P. and grant no. 275954 to M.S.N.).

\section{References}

Berry SD, Thompson RF. 1978. Prediction of learning rate from the hippocampal electroencephalogram. Science 200: 1298-1300.

Bragin A, Jando G, Nádasdy Z, Hetke J, Wise K, Buzsáki G. 1995. y (40-100 $\mathrm{Hz}$ ) Oscillation in the hippocampus of the behaving rat. J Neurosci 15: 47-60.

Buzsáki G. 1989. Two-stage model of memory trace formation: a role for "noisy" brain states. Neuroscience 31: 551-570.

Buzsáki G. 2002. $\theta$ oscillations in the hippocampus. Neuron 33: $325-340$.

Buzsáki G, Moser EI. 2013. Memory, navigation and $\theta$ rhythm in the hippocampal-entorhinal system. Nat Neurosci 16: 130-138.

Buzsáki G, Leung LW, Vanderwolf CH. 1983. Cellular bases of hippocampal EEG in the behaving rat. Brain Res 287: 139-171.

Chrobak JJ, Buzsáki G. 1996. High-frequency oscillations in the output networks of the hippocampal-entorhinal axis of the freely behaving rat. J Neurosci 16: 3056-3066.

Darling RD, Takatsuki K, Griffin AL, Berry SD. 2011. Eyeblink conditioning contingent on hippocampal $\theta$ enhances hippocampal and medial prefrontal responses. J Neurophysiol 105: 2213-2224.

Givens B. 1996. Stimulus-evoked resetting of the dentate $\theta$ rhythm: relation to working memory. Neuroreport 8: 159-163.

Gould TJ, Steinmetz JE. 1996. Changes in rabbit cerebellar cortical and interpositus nucleus activity during acquisition, extinction, and backward classical eyelid conditioning. Neurobiol Learn Mem 65: $17-34$.

Griffin AL, Asaka Y, Darling RD, Berry SD. 2004. $\theta$-contingent trial presentation accelerates learning rate and enhances hippocampal plasticity during trace eyeblink conditioning. Behav Neurosci 118: 403-411.

Hasselmo ME, Stern CE. 2014. $\theta$ rhythm and the encoding and retrieval of space and time. Neuroimage 85 Pt 2: 656-666.

Hasselmo ME, Bodelón C, Wyble BP. 2002. A proposed function for hippocampal $\theta$ rhythm: separate phases of encoding and retrieval enhance reversal of prior learning. Neural Comput 14: 793-817.

Holscher C, Anwyl R, Rowan MJ. 1997. Stimulation on the positive phase of hippocampal $\theta$ rhythm induces long-term potentiation that can be depotentiated by stimulation on the negative phase in area CA1 in vivo. J Neurosci 17: 6470-6477.

Huerta PT, Lisman JE. 1995. Bidirectional synaptic plasticity induced by a single burst during cholinergic $\theta$ oscillation in CA1 in vitro. Neuron 15: 1053-1063.

Hyman JM, Wyble BP, Goyal V, Rossi CA, Hasselmo ME. 2003. Stimulation in hippocampal region CA1 in behaving rats yields long-term potentiation when delivered to the peak of $\theta$ and long-term depression when delivered to the trough. J Neurosci $\mathbf{2 3}$ : $11725-11731$

Kamondi A, Acsady L, Wang XJ, Buzsáki G. 1998. $\theta$ oscillations in somata and dendrites of hippocampal pyramidal cells in vivo: activity-dependent phase-precession of action potentials. Hippocampus 8: $244-261$.

McCartney H, Johnson AD, Weil ZM, Givens B. 2004. $\theta$ Reset produces optimal conditions for long-term potentiation. Hippocampus 14: $684-687$.

Mitzdorf U. 1985. Current source-density method and application in cat cerebral cortex: investigation of evoked potentials and EEG phenomena. Physiol Rev 65: 37-100.

Nicholson C, Freeman JA. 1975. Theory of current source-density analysis and determination of conductivity tensor for anuran cerebellum. J Neurophysiol 38: 356-368.

Nokia MS, Wikgren J. 2014. Effects of hippocampal state-contingent trial presentation on hippocampus-dependent nonspatial classical conditioning and extinction. J Neurosci 34: 6003-6010.

Nokia MS, Penttonen M, Korhonen T, Wikgren J. 2009. Hippocampal $\theta$-band activity and trace eyeblink conditioning in rabbits. Behav Neurosci 123: 631-640.

Nokia MS, Penttonen M, Wikgren J. 2010. Hippocampal ripple-contingent training accelerates trace eyeblink conditioning and retards extinction in rabbits. J Neurosci 30: $11486-11492$.

Nokia MS, Mikkonen JE, Penttonen M, Wikgren J. 2012a. Disrupting neural activity related to awake-state sharp wave-ripple complexes prevents hippocampal learning. Front Behav Neurosci 6: 84. 
Nokia MS, Sisti HM, Choksi MR, Shors TJ. 2012b. Learning to learn: $\theta$ oscillations predict new learning, which enhances related learning and neurogenesis. PLoS One 7: e31375.

Palva S, Linkenkaer-Hansen K, Naatanen R, Palva JM. 2005. Early neural correlates of conscious somatosensory perception. J Neurosci 25: $5248-5258$

Prokasy WF. 1984. Presidential address, 1983. Psychophysiology 21: 1-13. Siegle JH, Wilson MA. 2014. Enhancement of encoding and retrieval functions through $\theta$ phase-specific manipulation of hippocampus. Elife 3: e03061.
Skaggs WE, McNaughton BL, Wilson MA, Barnes CA. 1996. $\theta$ phase precession in hippocampal neuronal populations and the compression of temporal sequences. Hippocampus 6: 149-172.

Weiss C, Disterhoft JF. 2011. Exploring prefrontal cortical memory mechanisms with eyeblink conditioning. Behav Neurosci 125: $318-326$.

Received January 15, 2015; accepted in revised form April 10, 2015. 


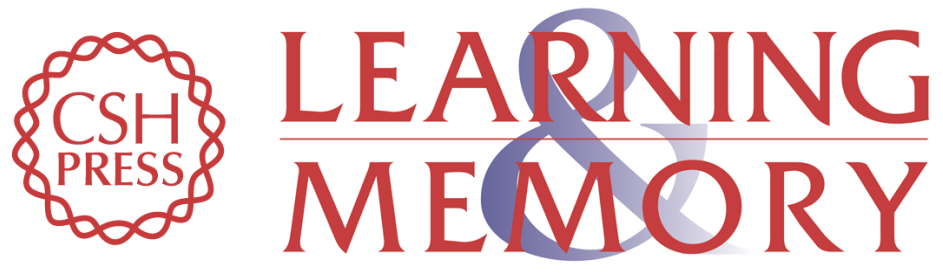

\section{Phase matters: responding to and learning about peripheral stimuli depends on hippocampal $\theta$ phase at stimulus onset}

Miriam S. Nokia, Tomi Waselius, Jarno E. Mikkonen, et al.

Learn. Mem. 2015, 22:

Access the most recent version at doi:10.1101/Im.038166.115

References

Creative Commons License

Email Alerting Service
This article cites 30 articles, 8 of which can be accessed free at: http://learnmem.cshlp.org/content/22/6/307.full.html\#ref-list-1

This article, published in Learning \& Memory, is available under a Creative Commons License (Attribution-NonCommercial 4.0 International), as described at http://creativecommons.org/licenses/by-nc/4.0/.

Receive free email alerts when new articles cite this article - sign up in the box at the top right corner of the article or click here. 